Sharif University of Technology
Scientia Iranica

\title{
Medium-term planning of vehicle-to-grid aggregators for providing frequency regulation service
}

\author{
M.H. Sarparandeh ${ }^{\mathrm{a}, *}$, M. Kazemi ${ }^{\mathrm{b}}$, and M. Ehsan ${ }^{\mathrm{a}}$ \\ a. Department of Electrical Engineering, Sharif University of Technology, Tehran, Iran. \\ b. Faculty of Electrical Engineering, University of Shahreza, Shahreza, Iran.
}

Received 7 May 2017; received in revised form 9 December 2017; accepted 26 June 2018

\author{
KEYWORDS \\ Electric vehicles; \\ Frequency regulation; \\ Pricing; \\ Vehicle-to-Grid \\ (V2G); \\ Aggregator.
}

\begin{abstract}
Utilization of electric vehicle battery to provide frequency regulation service in electricity markets is a technically feasible and economically attractive idea. The role of aggregators as a middleman between electric vehicle owners and the frequency regulation market has been discussed in the literature. However, the economic interaction between the aggregator and the vehicle owners on division of interests is still a missing point. In this paper, a new pricing model for aggregators of electric vehicles is proposed such that not only its profit is maximized, but also the vehicle owners have sufficient incentives to take part in the offered vehicle-to-grid program. In the proposed model, the aggregator takes into account the depreciation cost of electric vehicle batteries and the cost of net energy transaction between the electric vehicles and the grid and, then, considers these items in settling accounts with vehicle owners. The proposed model has been implemented on PJM frequency regulation market, and the results are discussed in the paper.
\end{abstract}

(C) 2020 Sharif University of Technology. All rights reserved.

\section{Introduction}

The application of Electric Vehicles (EVs) in transportation systems is a growing trend as they improve fuel consumption efficiency and reduce green-house gas emissions [1]. On the other hand, the decline of battery cost would decrease EVs cost. For example, based on a comparison of 2017 and 2015, it was predicted that battery cost would drop by $25 \%$ [2]. It was also estimated that EVs would cost less than 22,000\$ by 2040 [3]. In this way, a remarkable share for EVs in future automobile markets is predictable as stated in [4].

Along with the increasing number of EVs, the idea of utilizing $\mathrm{EV}$ batteries as distributed energy storage

\footnotetext{
*. Corresponding author.

E-mail address: sarparandeh@ee.sharif.edu. (M.H. Sarparandeh)
}

doi: $10.24200 /$ sci. 2018.20612 resources in electricity markets will be practicable. This service, which is known as vehicle-to-grid (V2G), has been defined as the power that can be fed to the grid by EVs through appropriate connections when the EVs are parked [5].

V2G can be applied to various electricity markets such as peak power, spinning reserves, and frequency regulation markets. Some cost-benefit studies have evaluated the profitability of $\mathrm{V} 2 \mathrm{G}$ for each market and have demonstrated that one of the most profitable markets for $\mathrm{V} 2 \mathrm{G}$ is the frequency regulation market $[5,6]$. Frequency regulation service is the use of fast response resources such as synchronized generators that have Automatic Generation Control (AGC) capability to keep the moment by moment balancing between generation and load. Regulation facilitates maintaining frequency under the proposed market arrangements and managing the differences between actual and scheduled power flows between balancing areas. The units that wish to participate 
in the regulation market must submit their bid to the system operator. Then, the system operator broadcasts AGC signal to the selected units in real time.

EV batteries are a suitable source for frequency regulation service due to their fast response in comparison with conventional thermal units [7]. However, the number of EVs is too huge for the power system operator to communicate with all of them directly. Furthermore, the minimum bid size requirements of current electricity markets are obstacles to direct participation. Hence, several papers in the literature have proposed a structure in which aggregators submit energy or regulation bids to markets on behalf of a fleet of EVs $[8,9]$. In this structure, the aggregators contract with interested EV owners and utilize their battery capacities according to some pre-specified conditions.

Although the technical and economic aspects of $\mathrm{V} 2 \mathrm{G}$ for providing frequency regulation service have been investigated in several pieces of research, proper pricing of this service is still a missing point in the literature. In other words, it is not clarified that how and how much the EV owners are paid by aggregators for sharing their batteries' capabilities. In [10], the profit from the participation of EVs in energy, reserve, and regulation markets was calculated; however, the share of the aggregator and EV owners from this profit was not determined. In [11], EVs optimize charging and regulation decisions, and the aggregator only sums the optimal decisions for each $\mathrm{EV}$ and submits bids to the Independent System Operator (ISO). It seems as if the aggregator is a nonprofit organization in [11], and its profit is not taken into account. In [12], the bidding strategy of an aggregator that participated in the day-ahead electrical energy market was addressed, ignoring the benefits of EV owners. A similar approach can be found in [13], where the profit of the aggregator was maximized through the optimal simultaneous bidding of $\mathrm{V} 2 \mathrm{G}$ energy and ancillary services, while the benefits of EV owners were not modeled, and the competition between different competing aggregators was ignored. In contrast, the problem considered in [14] assumes a hypothetical problem that a company owning a fleet of EVs schedules its EV fleet so as to optimize its battery charging, V2G revenue, and vehicles' duty/service. The problem was modeled as a multi-criteria decision-making problem and solved by analytic hierarchy process. However, the aggregator is assumed to be an exogenous player in [14], whose decisions are neglected. In [15], the aggregator maximized its profit from participation in the frequency regulation market while minimizing the charging costs of EVs. In [15], the EV owners were not paid for V2G service; thus, there was not enough incentive for $\mathrm{EV}$ owners to participate in $\mathrm{V} 2 \mathrm{G}$.

In this paper, the aggregator pays the $\mathrm{EV}$ owners for $\mathrm{V} 2 \mathrm{G}$ service according to the number of hours that they provide frequency regulation, taking into account EV batteries depreciation and the net electrical energy exchanged between the EV batteries and the grid. The price determination of $\mathrm{V} 2 \mathrm{G}$ service is a necessary step to evaluate whether there are sufficient incentives for both the EV owner and the aggregator to participate in such a cooperation. Hence, we have tried to model the pricing scheme of an aggregator for V2G service by maximizing the aggregator's profit and considering the economic behavior of $\mathrm{EV}$ owners.

The rest of the article is organized as follows. The pricing model with which the aggregator and the owners of EVs deal to cooperate on providing frequency regulation service via $\mathrm{V} 2 \mathrm{G}$ is proposed in Section 2 . Section 3 is devoted to a case study in which the V2G pricing model is utilized for frequency regulation as an ancillary service in PJM electricity market, and the results are presented in the same section. Finally, some remarks and directions for future work are discussed in Section 4.

\section{Pricing framework modeling}

This paper proposes medium-term planning for an EV aggregator to maximize its profit. The aggregator uses available battery capacities of its contracted EVs to participate in the frequency regulation market. In this way, the aggregator adjusts its strategy to participate in the regulation market and to interact with $\mathrm{EV}$ owners. The main decision variable of the aggregator in this process is its offered price to EV owners. In this section, the interactions between aggregators and the other participants are modeled, and a pricing scheme for $\mathrm{V} 2 \mathrm{G}$ is proposed.

\subsection{Model of interaction with EVs}

The aggregator offers the price to $\mathrm{EV}$ owners in exchange for their V2G service. This price-hereinafter referred to as $V 2 G$ price-will affect the number of EVs who are willing to sign on. If the $\mathrm{V} 2 \mathrm{G}$ price is very low, then no EV owner will agree on the contract. The higher is the price offered, more EV owners are willing to contract with the aggregator.

This procedure is similar to that of retailers' medium-term planning in which the offered price of retailer defines the number of its clients. Different methods have been proposed in the literature to address this problem from the retailer's perspective. In [16-18], the relation between the number of contracted customers and the offered retail price is introduced by Price-Quota-Curve (PQC). In this paper, in line with [16-18], a "V2G supply function" is proposed to model the relationship between $\mathrm{V} 2 \mathrm{G}$ price offered by the aggregator and the number of EVs who accept the $\mathrm{V} 2 \mathrm{G}$ program.

The V2G supply function provides the number of 


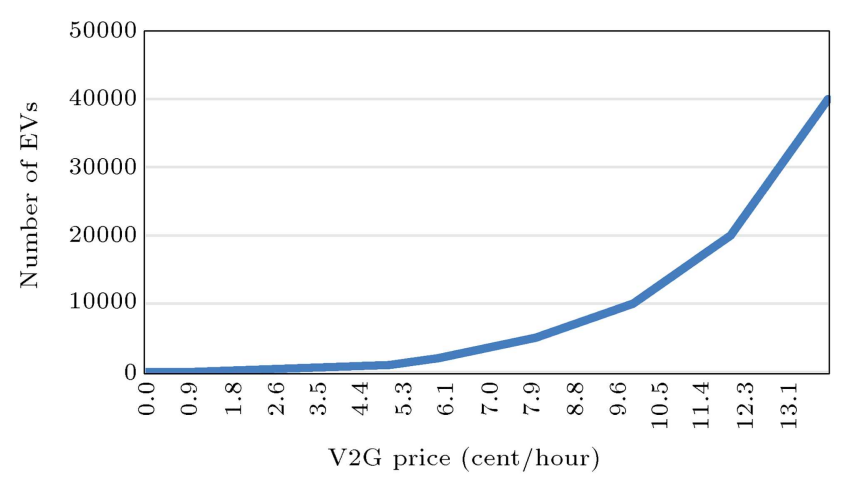

Figure 1. Vehicle-to-Grid (V2G) supply function.

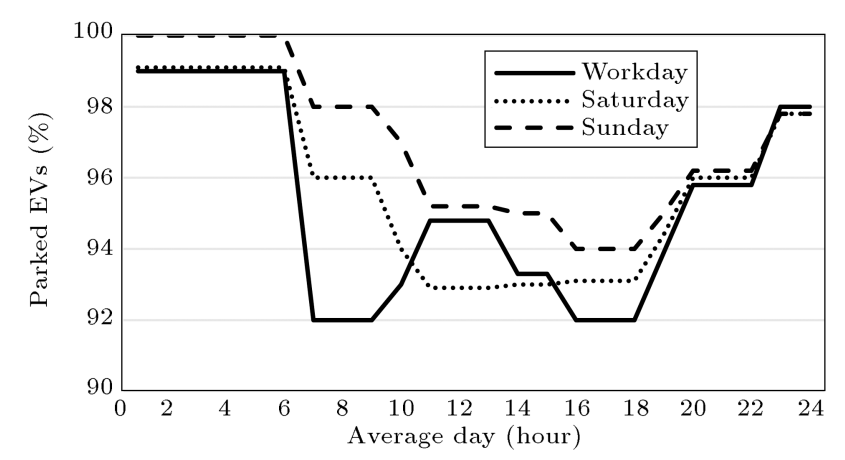

Figure 2. Share of parked Electric Vehicles (EVs) in dependence on day types [20].

EV owners that are willing to share their EV batteries in exchange for the offered V2G price. Figure 1 shows a piecewise linear PQC. The precise derivation of this function is an econometric problem, which is beyond the scope of this paper. Thus, the function has been considered as an input to our model and has been estimated by a hypothetical curve for the case study. However, the curve meets two important properties: it is increasing and its derivative is increasing, too. The latter can be explained by "diminishing marginal product": the property whereby the marginal product of an input declines as the quantity of the input increases [19]. Those EVs that are attracted to V2G contract earlier can provide $\mathrm{V} 2 \mathrm{G}$ service more hours of the day in comparison to busy EVs that may join subsequently.

Contracted EVs are not always parked and ready for V2G service. The share of available EVs can be estimated by statistical studies for different hours of the day and different day types, i.e., weekdays and weekends. In [20], the share of parked EVs was designated for different day types. Figure 2 displays the share of parked EVs for different hours and day types for a typical area.

In addition, it should be noted that not all parked EVs are available for V2G service. Only those EVs are available (a) that are parked in a place with necessary infrastructure (at least an outlet), (b) whose owners actually plugged-in the EV, and (c) whose batteries'
State-of-Charge (SoC) is proper enough to be used by the aggregator for the regulation service. In order to model the effect of the above-mentioned conditions, an "availability ratio" is used here. The availability ratio is defined as the ratio of available EVs ready for V2G to the number of parked EVs. In practice, the aggregator can determine the availability ratio of EVs based on its database record.

In order to guarantee the active participation and mutual benefit of aggregator and EVs, the quality of the interaction between the involved parties should be considered properly. For this purpose, three different types of payment are considered in this paper. In the first payment category, the aggregator pays the EV owners for every hour that the EVs are available for this service. In the second payment, at the end of each settlement period (e.g. a month), the net electrical energy received by each $\mathrm{EV}$ from the grid or injected to the grid by the EV will be metered and accounted in transactions between the aggregator and the EV owners. In the last one, the aggregator pays "battery depreciation cost" to the EV owners, which is a payment term in their invoice to compensate for their battery depreciation. These payments are discussed in the following sub-sections in detail.

\subsection{Model of interaction with regulation market}

The aggregator bids to the frequency regulation market for every hour according to the number of estimated available EVs. Nevertheless, the aggregator estimates have an error that is simulated in our model by a standard error. Hence, the number of real available EVs has been sampled for every hour randomly by a normal probability distribution function with the estimated number of available EVs as expected value and $5 \%$ of EVs as standard deviation. If the electric power of EVs' batteries is not sufficient to accomplish the frequency regulation service, the aggregator will operate a back-up battery bank.

The assumed regulation market structure of this paper is similar to the frequency regulation market of PJM. It is worth mentioning that the ancillary services that are used to balance the generation and consumption of electricity in real time (with little difference in different electricity markets, academic and technical literature) include frequency response, frequency regulation, spinning reserve, non-spinning reserve, and supplemental reserve. In PJM, frequency response is not a stand-alone ancillary service product. All resources that provide spinning reserve must be synchronized with the grid and must be frequency responsive. Spinning, non-spinning, and supplemental reserves are reserve capabilities that can be converted fully into energy or load, which can be removed from the system within 10 minutes of the request from 
the PJM dispatcher. Frequency regulation, which is our goal market, refers to the control action that is performed to correct for load changes that may cause the power system to operate above or below $60 \mathrm{~Hz}$. The response time of this service is 2 seconds, and its duration is 5 minutes.

The mechanism for procuring frequency regulation service in PJM regulation market is described in [21]. Regulation service providers must submit their offers to P JM market. Then, PJM utilizes these offers together with energy offers and resource schedules to determine the lowest cost alternative for these services through an optimization process. In this process, the Regulation Market Clearing Price (RMCP) for each hour of the operating day is determined [21].

Regulation resources will receive the following regulation signals in PJM [22]:

- Assigned regulation (AReg): This is the regulation quantity (MW), which is announced to the participants whose offers are accepted in the regulation market. This signal is static during each hour, yet is sent on a 10 -second scan rate;

- Regulation control signal (RegA): This signal is sent by PJM to frequency regulation resources in real time to modify their generation/consumption according to the signal value and their contract. This signal will be transmitted on a 2-second scan rate;

- Fast or dynamic regulation (RegD): As a special feature of PJM market, this signal, which has a function similar to RegA, is transmitted by PJM to frequency regulation resources. However, it is preferred to be utilized for energy storage devices, since this signal is short-term balanced around zero.

To clarify the differences between RegA and $\operatorname{RegD}$, it is worth mentioning that there are different types of regulation resources such as conventional power plants (e.g., combined cycle, hydroelectric, etc.), the energy storage resources (e.g., batteries, flywheels, EVs, etc.), and also dispatchable loads. Energy storage systems are more precise in tracking regulation signal than other regulation resources; however, they suffer from limited energy capacity. RegD signal has a mean value of zero that prevents energy storage resources from excess charge/discharge. RegA is a function of slow filter of Area Control Error (ACE) and can remain full raise or lower for extended periods, whereas RegD is a function of fast filter of ACE. Figure 3 represents the formation of these signals clearly [22].

It is worth mentioning that the implementation of the concept of $\mathrm{V} 2 \mathrm{G}$ in practice requires bidirectional communication infrastructure between the aggregator and the EVs-through which the aggregator receives the status of EVs and transmits the regulation signal to

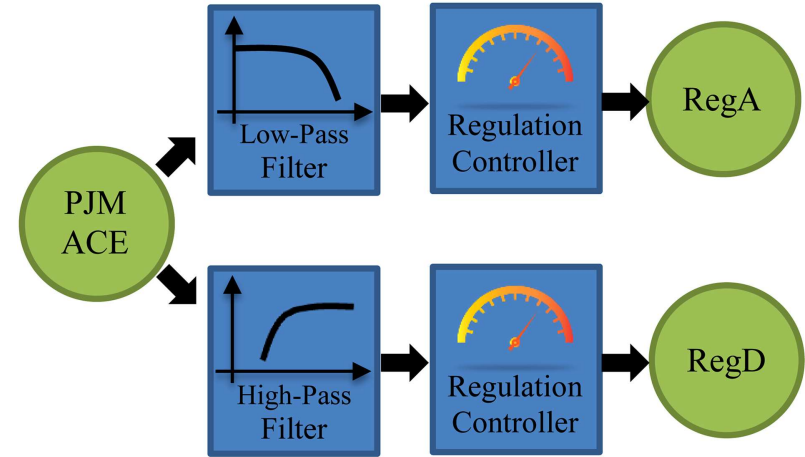

Figure 3. Formation of traditional regulation signal and fast (dynamic) regulation signal [22].

EVs. Thus, V2G can be realized after the widespread installation of smart meters and the development of smart grid. The pricing framework proposed in this paper presumes such infrastructure to be available.

\subsection{Model of $E V^{\prime}$ s battery degradation}

The effects of frequent charge/discharge cycles due to V2G on the lifetime of commercial Lithium-ion cells were discussed in [23-25]. The battery life is usually expressed as the number of cycles resulting in its capacity drop to a predetermined level (e.g., $80 \%$ of its initial capacity). It is shown that the achievable cycle count is a function of Depth-of-Discharge (DoD). Figure 4 illustrates the battery lifetime in cycles versus DoD [26]. As is shown in Figure 4, battery cycle life is related to the DoD. In other words, shallow cycling has much less impact on battery lifetime than deep cycling. Since frequency regulation causes shallow type of cycling, the battery degradation is not much worrying. Nevertheless, the lack of compensation for it may be frustrating for EV owners.

The curve depicted in Figure 4 has been estimated by Eq. (1) based on the approach of Rosenkranz [27] and the Fraunhofer ISE's model, as stated in [25] and [26]:

$$
N_{\text {Cycles }}=1331 \times\left(D o D_{V 2 G}\right)^{-1.8248},
$$

where $N_{\text {Cycles }}$ is the expected battery lifetime in terms

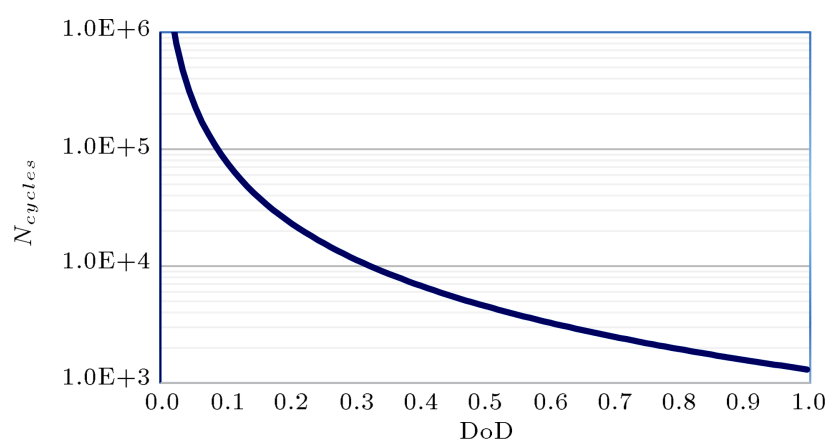

Figure 4. Estimated battery lifetime in cycles as a function of Depth-of-Discharge (DoD) [26]. 
of cycles, and $D o D_{V 2 G}$ is the DoD of EV battery for providing regulation service. By assuming a fixed cycling pattern, $D o D_{V 2 G}$ can be determined through the following equation.

$$
D_{o} D_{V 2 G}=P_{c h} \times T_{c h} / E_{b a t},
$$

where $P_{c h}$ is the charge/discharge power in $\mathrm{kW}, T_{c h}$ is the charge/discharge duration in hour, and $E_{b a t}$ is the capacity of battery in $\mathrm{kWh}$.

Due to the unknown cycling pattern in tracking regulation signal, expressing battery lifespan in terms of its energy throughput would be more practical. The energy throughput is a parameter that specifies the transacted energy of a battery in both charge and discharge modes over its whole lifetime. This parameter can be calculated by the following equation [24]:

$$
E_{E T}=N_{C y c l e s} \times D o D_{V 2 G} \times E_{b a t} .
$$

In the above equation, $E_{E T}$ is the lifetime energy throughput of the battery in $\mathrm{kWh}$. In this way, the "battery depreciation cost" can be obtained through Eq. (4):

$$
C_{d e p}=E_{b a t} \times \pi_{b a t} \times E_{T} / E_{E T},
$$

where $C_{d e p}$ is the battery depreciation cost during a certain period in $\$, \pi_{b a t}$ is the battery price in $\$ / \mathrm{kWh}$, and $E_{T}$ is the total transferred energy during the same period in $\mathrm{kWh}$.

\subsection{Proposed pricing model}

The proposed pricing scheme for $\mathrm{V} 2 \mathrm{G}$ service can be described by an optimization problem that maximizes the aggregator's profit and considers the behavior of the EV owners through V2G supply function. The objective function is described in Eq. (5):

$$
\begin{aligned}
\max _{\pi_{V 2 G}} \Omega_{T}= & \Sigma_{t=1}^{T} \pi_{f r}(t) \times N_{e s t}(t) \times P_{E V}-\Sigma_{t=1}^{T} \\
& N_{\text {real }}(t) \times \pi_{V 2 G}-E_{\text {net }} \times \pi_{r}-C_{\text {dep }, E V} \\
& -C_{d e p, b b},
\end{aligned}
$$

where $\Omega_{T}$ is the profit of aggregator during the simulation time span in $\$$. The main decision variable of this optimization problem is $\pi_{V 2 G}$, which is offered by the aggregator to EV owners in exchange for using their battery for regulation service. The objective has 4 terms. The first one $\Sigma_{(t=1)}^{T} \pi_{f r}(t) \times N_{e s t}(t) \times P_{E V}$ models the revenue gained from participating in the regulation market in which $\pi_{f r}(t)$ is the frequency regulation price of the electricity market in hour $t$ in $\$ / \mathrm{kWh}, P_{E V}$ is the rated charge/discharge power of $\mathrm{EV}$ batteries in $\mathrm{kW}$, and $N_{\text {est }}(t)$ is the estimated number of EVs in hour $t$ who are ready to provide regulation service, which is obtained by V2G supply function. The second term, i.e., $\Sigma_{t=1}^{T} N_{\text {real }}(t) \times \pi_{V 2 G}$, models the payment of the aggregator to $\mathrm{EV}$ owners for participation in $\mathrm{V} 2 \mathrm{G}$ program. In this term, $\pi_{V 2 G}$ is $\mathrm{V} 2 \mathrm{G}$ price that the aggregator offers to pay EV owners in $\$ / \mathrm{h}$, and $N_{\text {real }}(t)$ is the real number of EVs in hour $t$ who are ready to provide regulation service, which is simulated in our model by adding a standard error to $N_{\text {est }}(t)$. The third term in Eq. (5) models the payment to EV owners for the net energy delivered by them to the grid, where $\pi_{r}$ is the retail electricity price in $\$ / \mathrm{kWh}$, and $E_{\text {net }}$ is the sum of net energy transferred to/from grid by $\mathrm{EV}$ batteries over simulation time span in kWh. Finally, $C_{d e p, E V}$ and $C_{d e p, b b}$ are battery depreciation cost in $\$$ for whole EV batteries and backup battery bank, respectively, which are calculated by Eq. (4).

The capacity offered to the regulation market is $N_{e s t}(t) \times P_{E V}$ based on the explanations of Eq. (5). However, a ratio of this capacity is utilized by the regulation market in each 2 -second time step, which is determined by the RegD signal (see Section 2.2). In this way, the transacted power in the regulation market can be evaluated using Eq. (6).

$$
P_{\operatorname{Reg}}(\tau)=\operatorname{Reg} D(\tau) \times N_{e s t}(t) \times P_{E V},
$$

where $P_{R e g}(\tau)$ is the power that must be delivered to/absorbed from the grid by the aggregator in time step $\tau$ in $\mathrm{kW}, \operatorname{Reg} D(\tau)$ is the value of the frequency regulation signal sent by the power system operator to the aggregator in time step $\tau$, which is $-1<$ $\operatorname{Reg} D(\tau)<1$. In the proposed model, $P_{\operatorname{Reg}}(\tau)$ can be provided by the $\mathrm{EV}$ batteries as the first option or the backup battery bank as the second option. The shares of EV batteries and the backup battery in providing regulation power are determined by $P_{E V f}(\tau)$ and $P_{b b b}(\tau)$ through Eqs. (7) and (8), respectively.

$$
\begin{aligned}
& P_{E V f}(\tau)= \\
& \begin{cases}N_{\text {real }}(t) \times P_{E V} & \text { if } \quad P_{\text {Reg }}(\tau)>N_{\text {real }}(t) \times P_{E V} \\
-N_{\text {real }}(t) \times P_{E V} & \text { if } \quad P_{\text {Reg }}(\tau)>-N_{\text {real }}(t) \times P_{E V} \\
P_{\text {Reg }}(\tau) & \text { otherwise }\end{cases} \\
& P_{b b}(\tau)=\left\{\begin{array}{c}
\min \left\{P_{\text {Reg }}(\tau)-P_{E V f}(\tau), P_{b b, r}\right\} \\
\text { if } \quad P_{\text {Reg }}(\tau)>P_{E V f}(\tau) \\
0 \quad \text { if } \quad P_{\operatorname{Reg}}(\tau)=P_{E V f}(\tau) \\
\max \left\{P_{\operatorname{Reg}}(\tau)-P_{E V f}(\tau),-P_{b b, r}\right\} \\
\text { if } \quad P_{\operatorname{Reg}}(\tau)<P_{E V f}(\tau)
\end{array}\right.
\end{aligned}
$$

In the above equations, $P_{E V f}(\tau)$ and $P_{b b}(\tau)$ are the powers of EV fleet and back-up battery bank in time step $\tau$ in $\mathrm{kW}$, respectively, and $P_{b b, r}$ is the rated power of back-up battery bank in $\mathrm{kW}$. The 
batteries deliver electricity to the grid when $P_{E V f}(\tau)$ and $P_{b b}(\tau)$ are positive, and charge when these powers are negative.

The performance of the aggregator in providing frequency regulation service will be poor if it fails to follow $P_{\text {Reg }}(\tau)$ completely due to the shortage of EV fleet and back-up battery bank constraints. Different markets adopt different methods for calculating the performance of frequency regulation service providers. However, the performance depends on the difference between the regulation service actually provided and the regulation supposed to be provided. This quantity can be calculated by Eq. (9):

$$
P_{d i f f}(\tau)= \begin{cases}P_{b b}^{*}(\tau)-P_{b b, r} & \text { if } \quad P_{b b}^{*}(\tau)>P_{b b, r} \\ -P_{b b}^{*}(\tau)-P_{b b, r} & \text { if } \quad P_{b b}^{*}(\tau)<-P_{b b, r} \\ 0 & \text { otherwise }\end{cases}
$$

where $P_{b b}^{*}(\tau)$ is the power of back-up battery bank in time step $\tau$, provided that the capacity of back-up battery bank is unlimited. $P_{b b}^{*}(\tau)$ will be obtained by Eq. (8), if $P_{b b, r}$ is infinite.

The SoC of the back-up battery bank will be changed after any charge/discharge. Its value in any time step is dependent on its previous value as described in Eq. (10) for a 2-second frequency regulation time step:

$$
\begin{aligned}
& S_{o} C_{b b}(\tau)= \\
& \left\{\begin{array}{lll}
S o C_{b b}(\tau-1)-\frac{P_{b b}(\tau)}{E_{b b} \times \eta_{d i s} \times 1800} & \text { if } & P_{b b}(\tau) \geq 0 \\
S o C_{b b}(\tau-1)-\frac{P_{b b}(\tau) \times \eta_{c h}}{E_{b b} \times 1800} & \text { if } & P_{b b}(\tau)<0(10)
\end{array}\right.
\end{aligned}
$$

where $S o C_{b b}(\tau)$ is the state of charge of back-up battery bank in time step $\tau, E_{b b}$ is the capacity of back-up battery bank in $\mathrm{kWh}$, and $\eta_{c h}$ and $\eta_{\text {dis }}$ are charging and discharging efficiency of the battery, respectively. For a 2-second frequency regulation time step, an hour consists of 1800 time steps.

A similar equation can be considered for $\mathrm{EV}$ batteries by substituting the power and capacity of the EV battery into the parameters of the back-up battery bank. If the resultant SoC from Eq. (10) is outside the range of zero to one, the following modification must be performed:

$$
\begin{aligned}
& \text { If } S_{o C_{b b}(\tau)>1} \\
& \qquad \begin{array}{l}
P_{d i f f}^{m}(\tau)=P_{d i f f}(\tau)+E_{b b}\left(S o C_{b b}(\tau)-1\right) \times 1800 \\
P_{b b}^{m}(\tau)=P_{b b}(\tau)+E_{b b}\left(S o C_{b b}(\tau)-1\right) \times 1800 \\
S o C_{b b}^{m}(\tau)=1
\end{array}
\end{aligned}
$$

$$
\begin{aligned}
& \text { If } S o C_{b b}(\tau)<0, \\
& \qquad\left\{\begin{array}{l}
P_{d i f f}^{m}(\tau)=P_{d i f f}(\tau)-E_{b b} \times S o C_{b b}(\tau) \times 1800 \\
P_{b b}^{m}(\tau)=P_{b b}(\tau)-E_{b b} \times S o C_{b b}(\tau) \times 1800 \\
S o C_{b b}^{m}(\tau)=0
\end{array}\right.
\end{aligned}
$$

In the above equations, variables with superscript of $m$ are modified variables.

We have considered the average ratio of $P_{\text {diff }}(\tau)$ to $P_{\text {Reg }}(\tau)$ during simulation time span as the performance criteria, and the cases with poor performance are eliminated from solutions.

As the last equation, the total transferred energy of back-up battery bank during the time span of simulation can be obtained through Eq. (13) as follows:

$$
E_{T, b b}=\Sigma_{\tau=1}^{T}\left|P_{b b}(\tau)\right| / 1800,
$$

where $E_{T, b b}$ is the total transferred energy of back-up battery bank during $T$. A similar equation can be considered for EV batteries by replacing $P_{b b}(\tau)$ with $P_{E V f}(\tau) / N_{\text {real }}(t)$.

The planning horizon is set to be one year, and the aggregator can update the V2G price annually. Since the time steps in frequency regulation service are to the extent of few seconds, the simulation time span is reduced to one month, i.e., a financial settlement period between the aggregator and the EV owners, as the representative of twelve months of the year so as to decrease the solution time. In addition, the profit of the aggregator is a complicated function of the V2G price with many conditional functions. Hence, the problem has been optimized by an evolutionary optimization method. The genetic algorithm, which is used in this paper, is one of the evolutionary algorithms that is most commonly applied to solve combinatorial optimization problems.

Figure 5 displays the flowchart of our pricing framework. As can be seen in Figure 5, the aggregator first sets a V2G price to be offered to the EV owners. Then, the number of EVs that are eager to participate in frequency regulation service and also the number of available EVs for every hour of the day can be estimated. During the simulation time span, the AGC signal is transmitted from the power system operator to the aggregator, while the aggregator utilizes the batteries of available EVs to fulfill its obligations towards the power market. In case of lack of capacity to provide frequency regulation service, the back-up battery bank will be operated.

At the end of each simulation time span, the following required parameters are obtained: total transferred energy of EV batteries and the backup battery bank, the number of hours of availability for 


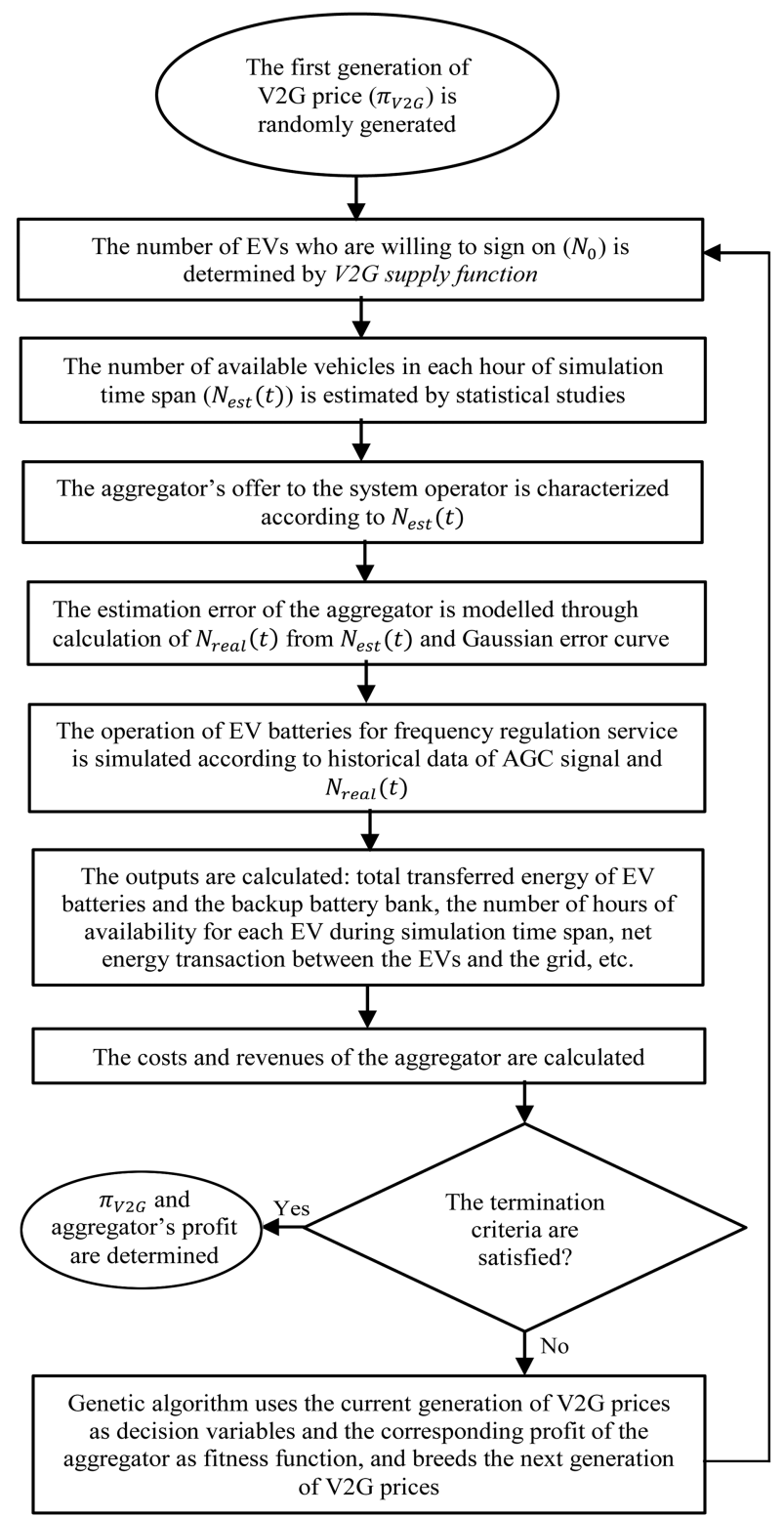

Figure 5. Flowchart of the proposed pricing framework.

each EV during simulation time span, and net energy transaction between the EVs and the grid. Then, the costs, revenues, and finally the profit of aggregator for each V2G price are calculated. Genetic algorithm considers the current generation of $\mathrm{V} 2 \mathrm{G}$ prices as decision variables and the corresponding profit of the aggregator as fitness values to generate the next generation of $\mathrm{V} 2 \mathrm{G}$ prices so that the profit of aggregator can be improved. This iterative algorithm continues until the termination criteria are satisfied.

\section{Case study and results}

This study applied the pricing framework proposed in the previous section to the frequency regulation market of The PJM-one of the world's largest competitive wholesale electricity markets. First, the input data of the case study are introduced; then, the results are presented.

\subsection{Input data and optimization parameters}

The pricing model described in Section 2 and depicted by Figure 5 is implemented on P JM electricity market. In our case study, the $\mathrm{V} 2 \mathrm{G}$ price offered by the aggregator to $\mathrm{EV}$ owners is determined so that the aggregator profit is maximized in a time span of one month. RMCP and RegD signal have been derived from real historical data of PJM for a one month period. Utilization of real historical data is a proper way to consider the uncertainty and random nature of changing RMCP, volatile RegD signal, and uncertain number of available EVs in each hour. A one-month period includes $31 \times 24$ hours, and each has different parameters and conditions. In the case of RegD signal that has a variant quantity in every two-second period, using its real historical data in a one-month period will consider the signal's stochastic nature, too. Therefore, the real historical data have been applied to model the random changes and the uncertainty of these parameters as an alternative for probability distribution functions used in stochastic methods.

The RMCP of PJM electricity market on 1 January 2013 is depicted in Figure 6 [28]. The RMCP is the sum of the regulation market capability clearing price and the regulation market performance clearing price [29]. The price at which the aggregator settles the net electrical energy flow between an EV and the grid, i.e., $\pi_{r}$, is set to 12 cents per $\mathrm{kWh}$. This value is chosen in a way that is close to the average retail electricity prices for residential customers across PJM region. RegD signal in January 2013 is obtained from PJM website whose changes for a one-hour period are plotted in Figure 7 [30].

As discussed in Section 2, "V2G supply function" has been considered by a hypothetical curve illustrated in Figure 1. In addition, data of [20] are used to define the share of parked EVs for different types of the day (please see Figure 2), and the availability

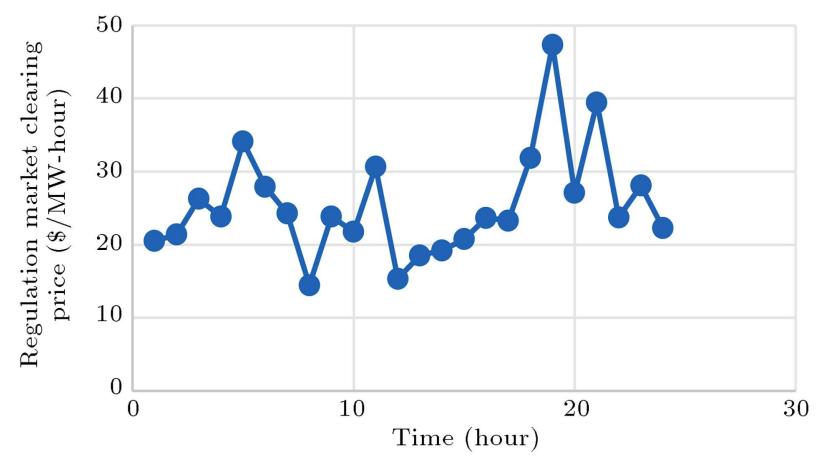

Figure 6. PJM regulation market clearing price (1 January 2013) [28]. 


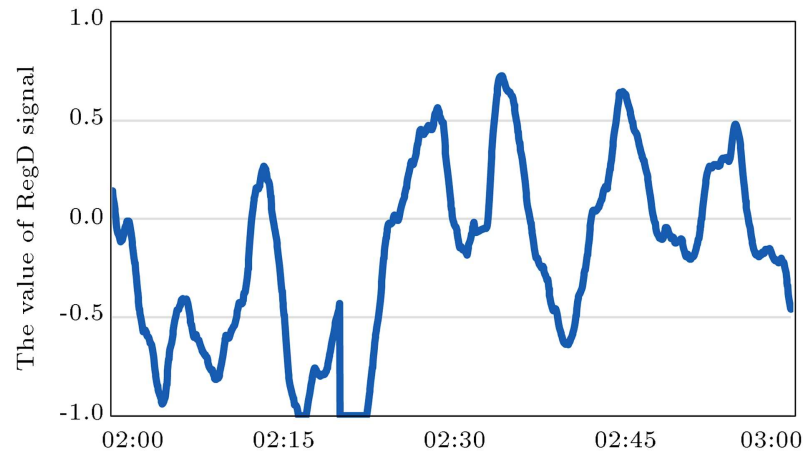

Figure 7. RegD signal in (PJM) (1 January 2013, 02:00 03:00 AM) [30].

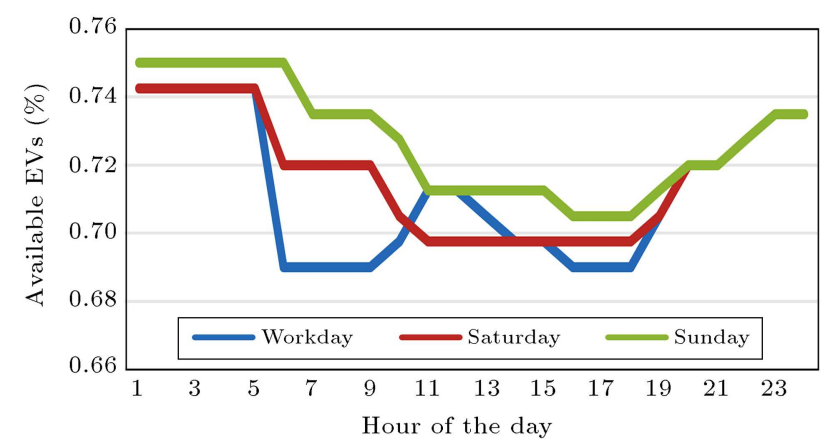

Figure 8. Availability share of Electric Vehicles (EVs) that have a contract with the aggregator.

ratio is assumed to be 0.75 . By assuming the abovementioned data, the available $\mathrm{EVs}$ are presented in Figure 8.

Assuming that the EVs are of plug-in type and that they are connected to the grid through household sockets, it is reasonable to consider the size of $3 \mathrm{~kW}$ for rating power electronics converter-which is an interface between the EV battery and the grid. The capacity of EV batteries is supposed to be similar and equal to $20 \mathrm{kWh}$, although as will be seen later, frequency regulation service following RegD signal does not affect the SoC of EV batteries significantly. Therefore, the capacity of EV batteries does not have a remarkable impact on our model. Both charging efficiency $\left(\eta_{c h}\right)$ and discharging efficiency $\left(\eta_{d i s}\right)$ of EV batteries are considered to be 0.95 as in [31], resulting in roundtrip efficiency of approximately 0.9 . The capacity of the back-up battery bank is considered to be fixed and equal to $1000 \mathrm{kWh}$, and the converter rated power is equal to $200 \mathrm{~kW}$. The initial SoC for back-up battery bank and all EVs is assumed to be $50 \%$.

As is stated in [23], $D o D_{V 2 G}$ is considered to be $3 \%$ in some papers, resulting in $10^{6}$ cycles. It is a rational approximation for our case, because, during January 2013, we could not find a period longer than 10 minutes in which regulation signal (RegD) had similar signs for all 2-second time steps. Therefore, for an EV battery with $20 \mathrm{kWh}$ capacity and $3 \mathrm{~kW}$ charge/discharge power, a maximum of $2.5 \%$ DoD is feasible according to Eq. (2).

The battery price of Lithium-ion batteries is declining and has fallen from 500-550 \$/kWh in 2014 to less than $400 \$ / \mathrm{kWh}$ in 2017 and, based on some assertions, even to a surprising value of $200 \$ / \mathrm{kWh}$ [32]. A battery price of $350 \$ / \mathrm{kWh}$ has been concluded as a result of various declared prices for our case study.

The pricing model has been optimized by NSGAII algorithm in MATLAB 7.12.0. NSGA-II is a controlled elitist genetic algorithm. Since it is elitist, it prefers solutions with a better fitness value. As it is controlled, it also favors the solutions that increase the diversity of the population. Diversity of population helps the algorithm converge to the optimal solution. The population size of each generation in NSGA-II algorithm is set to 20 individuals. In order to ensure appropriate performance of the aggregator in providing frequency regulation, the cases where the average ratio of $P_{\text {diff }}(\tau)$ to $P_{\text {Reg }}(\tau)$ during simulation time span is more than $1 \%$ are eliminated from solutions. The algorithm termination criteria include the combination of the maximum number of generations, time constraints, and lack of significant improvement in fitness values.

\subsection{Results}

By using the mentioned data of the previous subsection, the proposed algorithm of Figure 5 is applicable. The optimum level of $\mathrm{V} 2 \mathrm{G}$ price is 3.81 cents per hour. This price will attract $702 \mathrm{EV}$ owners to reach an agreement with the aggregator to provide V2G service for frequency regulation market. The economic results for various $\mathrm{V} 2 \mathrm{G}$ price scenarios are shown in Table 1. The value of the objective function is declared in this table term by term. As can be seen in this table, for $\mathrm{V} 2 \mathrm{G}$ prices higher than 7 cents per hour, the aggregator will lose due to high payments to $\mathrm{EV}$ owners; meanwhile, the EV owners do not have enough motivation to participate in $\mathrm{V} 2 \mathrm{G}$ service for prices less than 1 cent per hour.

Based on Eqs. (7) and (8), when the capacity of $\mathrm{EV}$ batteries is lower than the requested power by the frequency regulation market, the capacity of backup battery is used. Figures 9 and 10 display the transacted power between the EV fleet and the grid and that between back-up battery bank and the grid for a period of one hour, respectively.

The results of analyzing the charge/discharge pattern of the back-up battery bank during our monthly period show that this battery bank has been rarely utilized, because the prediction error of the available $\mathrm{EVs}$ is assumed to be low. Because of this fact, the SoC of the back-up battery bank will not approach its boundary limits. For more clarification, the power and the SoC of the backup battery are presented for the whole January in Figures 11 and 12, respectively. 
Table 1. Economic results of various Vehicle-to-Grid (V2G) price scenarios in a 1-month period.

\begin{tabular}{cccccccc}
\hline $\begin{array}{c}\text { V2G Price } \\
\text { (cent/hour) }\end{array}$ & $\begin{array}{c}\text { No. of } \\
\text { EVs }\end{array}$ & $\begin{array}{c}\text { Revenue of } \\
\text { aggregator } \\
(\$)\end{array}$ & $\begin{array}{c}\text { Back-up } \\
\text { batteries } \\
\text { degradation } \\
\text { cost }(\$)\end{array}$ & $\begin{array}{c}\text { EV fleet } \\
\text { batteries } \\
\text { degradation } \\
\text { cost }(\$)\end{array}$ & $\begin{array}{c}\text { Payment for } \\
\text { net transacted } \\
\text { power }(\$)\end{array}$ & $\begin{array}{c}\text { Payment for } \\
\text { V2G to EV }\end{array}$ & $\begin{array}{c}\text { Profit of } \\
\text { oggregator }\end{array}$ \\
\hline $\mathbf{2}$ & 250 & 13521 & 3 & 975 & 1810 & 3488 & $\mathbf{7 2 4 6}$ \\
$\mathbf{( \$ )}$
\end{tabular}

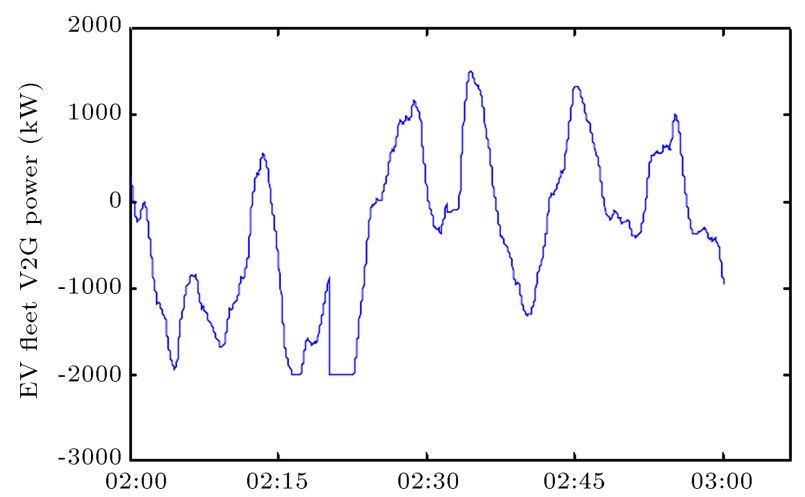

Figure 9. Electric Vehicle (EV) fleet batteries power during January 1, 02:00 to 03:00 AM.

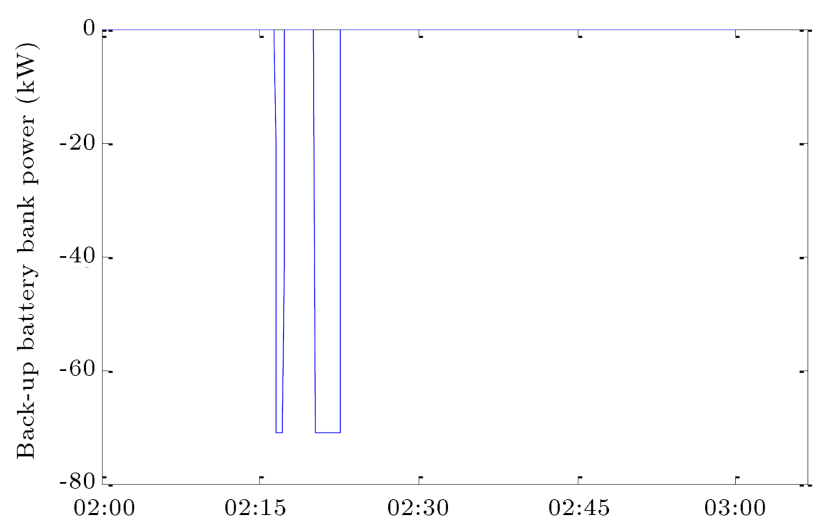

Figure 10. Back-up batteries power during January 1, 02:00 to 03:00 AM.

From the aggregator's point of view, the monthly profit is $11,532 \$$, which means more than $135,000 \$$ profit in a year. Moreover, an EV owner whose vehicle is available $60 \%$ of the day on average for $\mathrm{V} 2 \mathrm{G}$ service will receive more than $200 \$$ per year. If the cost of required communication and control infrastructure for V2G implementation is considered to be about $1000 \$$

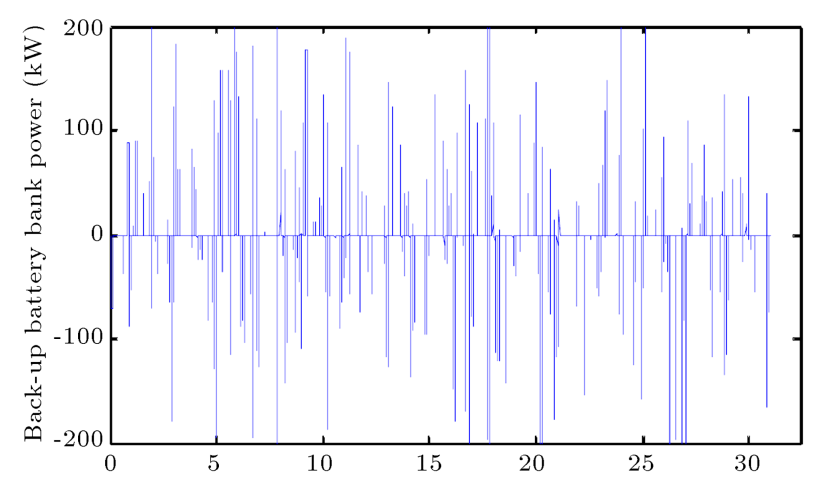

Figure 11. Back-up battery bank power during January.

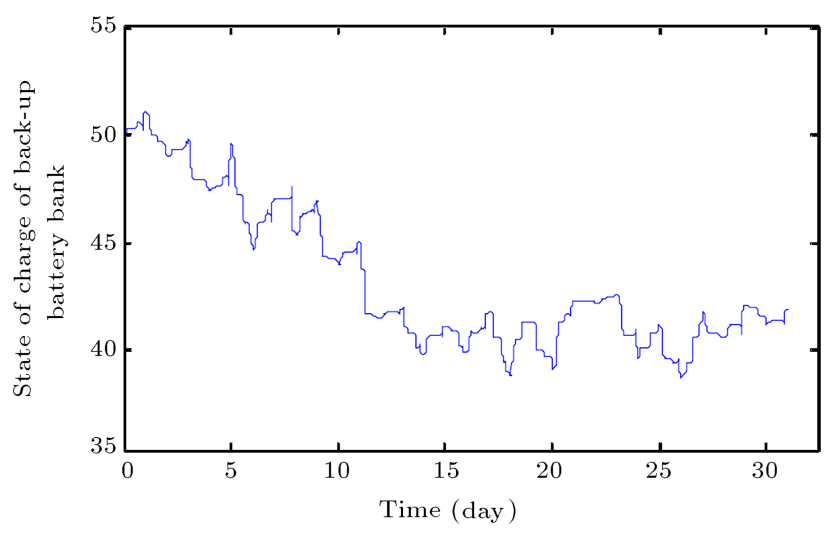

Figure 12. Back-up battery bank state of charge during January.

per vehicle, the payback period to recoup the funds expended for required infrastructure will be 5 years. Based on what we have proposed, the aggregator transfers this fund to EV owners. Because, in this case, the EVs with maximum availability time at home have the motivation to participate in V2G service. However, the aggregator may pay this initial cost, and the EV owners pay it by installments through their income from V2G. 


\section{Conclusion}

Implementation of Vehicle-to-Grid (V2G) concept requires active participation of EV owners. In this paper, a novel pricing model was proposed to determine the price that an aggregator should pay EV owners in order to encourage them to take part in providing frequency regulation service, while the profit of the aggregator is maximized. The proposed pricing model was applied to PJM frequency regulation market, considering fast regulation ( $\mathrm{RegD}$ ) signal. The results indicated that the depth of discharge for both EV batteries and the back-up batteries was insignificant enough to ensure numerous life cycles for the batteries. In addition, the SoC of the back-up battery bank would not approach the fully charged/discharged mode. Moreover, the fast regulation signal used in our case had a mean value of zero, which has a negligible impact on SoC of EV batteries. Therefore, EV owners would not be concerned about driving with empty battery in the hours ahead.

The paper comes to the following findings:

- A model was presented for the interaction between the aggregators and EV owners that considered the interests of both parties and motivated them to participate in $\mathrm{V} 2 \mathrm{G}$;

- A pricing scheme was introduced to maximize the profit of aggregators;

- If the V2G supply function was obtained precisely, the exact profit of EV owners and aggregators could be determined so as to evaluate the economic attractiveness of $\mathrm{V} 2 \mathrm{G}$.

In the future works, the capacity of back-up battery bank can be considered as a variable to be optimized as the second objective in addition to $\mathrm{V} 2 \mathrm{G}$ price. In addition, the economic risk of the aggregator can be considered as an independent objective. Furthermore, the aggregator planning problem can be dealt with as a multi-criteria decision-making problem.

\section{References}

1. Khan, S. and Kushler, A., Plug-in Electric Vehicles: Challenges and Opportunities, American Council for an Energy-Efficient Economy (2013). Available at: http://www.aceee.org/research-report/t133.

2. Maloney, P. "After record year, U.S. energy storage forecasted to break $1 \mathrm{GW}$ capacity mark in 2019", Utility Dive, U.S. (2016). Available at: http://www.utilitydive.com/news/after-record-yearus-energy-storage-forecasted-t o-break-1-gw-capacityma/415081/, accessed Dec. 2016.

3. DeBord, M. "Electric cars could be nearing a critically important tipping point for explosive growth", Business Insider (2016). Available at: http://www.businessinsider.com/the-2020s-could-bethe-decade-of-the-electric-car-2016-2 accessed Jan. 2017.

4. "World Energy Outlook", International Energy Agency (2011). Available at: http://www.iea.org/publications/freepublications/ publication/WEO2011-WEB.pdf.

5. Kempton, W. and Tomic, J. "Vehicle-to-grid power implementation: From stabilizing the grid to supporting large-scale renewable energy", Journal of Power Sources, 144, pp. 280-294 (2005).

6. Hosseini, S.S., Badri, A., and Parvania, M. "A survey on mobile energy storage systems (MESS): applications, challenges and solutions", Renewable \& Sustainable Energy Reviews, 40, pp. 161-170 (2014).

7. Vagropoulos, S.I. and Bakirtzis, A.G. "Optimal bidding strategy for electric vehicle aggregators in electricity markets", IEEE Transactions on Power Systems, 28(4), pp. 4031-4041 (2013).

8. Guille, C. and Gross, G. "A conceptual framework for the vehicle-to-grid (V2G) implementation", Energy Policy, 37, pp. 4379-4390 (2009).

9. Quinn, C., Zimmerle, D., and Bradley, T.H. "The effect of communication architecture on the availability, reliability, and economics of plug-in hybrid electric vehicle-to-grid ancillary services", Journal of Power Sources, 195(5), pp. 1500-1509 (2010).

10. Kempton, W., Udo V., Huber, K., Komara, K., Letendre, S., Baker, S., Brunner, D., and Pearre, N., A Test of Vehicle-to-Grid (V2G) for Energy Storage and Frequency Regulation in the PJM System, University of Delaware, Pepco Holdings, PJM Interconnect, Green Mountain College (2008).

11. Donadee, J. and Ilić, M. "Stochastic co-optimization of charging and frequency regulation by electric vehicles", 2012 North American Power Symposium (NAPS), Champaign, IL, pp. 1-6 (2012).

12. Baringo, L. and Amaro, R.S. "A stochastic robust optimization approach for the bidding strategy of an electric vehicle aggregator", Electric Power Systems Research, 146, pp. 362-370 (2017).

13. Sortomme, E. and El-Sharkawi, M.A. "Optimal scheduling of vehicle-to-grid energy and ancillary services", IEEE Transactions on Smart Grid, 3(1), pp. 351-359 (2012).

14. Janjic, A. and Velimirovic, L.Z. "Optimal scheduling of utility electric vehicle fleet offering ancillary services", ETRI Journal, 37(2), pp. 273-282 (2015).

15. Sortomme, E. and El-Sharkawi, M.A. "Optimal charging strategies for unidirectional vehicle-to-grid", IEEE Transactions on Smart Grid, 2, pp. 131-138 (2011).

16. Gabriel, S.A., Conejo, A.J., Plazas, M.A., and Balakrishnan, S. "Optimal price and quantity determination for retail electric power contracts", IEEE Transactions on Power Systems, 21(1), pp. 180-187 (2006). 
17. Carrion, M., Conejo, A.J., and Arroyo, J. M. "Forward contracting and selling price determination for a retailer", IEEE Transactions on Power Systems, 22(4), pp. 2105-2114 (2007).

18. Kharrati, S., Kazemi, M., and Ehsan, M. "Mediumterm retailer's planning and participation strategy considering electricity market uncertainties", Int. Trans. Electr. Energ. Syst., 26, pp. 920-933 (2016).

19. Mankiw, N.G., Principles of Microeconomics, Thomson Nelson, Toronto, CA (2008).

20. Fluhr, J., Ahlert, K.H., and Weinhardt, C. "A stochastic model for simulating the availability of electric vehicles for services to the power grid", $201043 r d$ Hawaii International Conference on System Sciences, Honolulu, HI, pp. 1-10 (2010).

21. Pandurangan, V., Zareipour, H., and Malik, O. "Frequency regulation services: A comparative study of select North American and European reserve markets", North American Power Symposium (NAPS), Champaign, IL, pp. 1-8 (2012).

22. "Ancillary Services", PJM State \& Member Training Department (2013). Available at:

http://www.pjm.com//media/training/corecurriculum/ip-gen-301/gen-301-ancillaryservices.ashx, accessed February 2013.

23. Hill, D.M., Agarwal, A.S., and Ayello, F. "Fleet operator risks for using fleets for V2G regulation", Energy Policy, 41, pp. 221-231 (2012).

24. Bishop, J.D., Axon, C.J., Bonilla, D., Tran, M., Banister, D., and McCulloch, M.D. "Evaluating the impact of V2G services on the degradation of batteries in PHEV and EV", Applied Energy, 111, pp. 206-218 (2013).

25. Dallinger, D., Krampe, D., and Wietschel, M. "Vehicle-to-grid regulation reserves rased on a dynamic simulation of mobility behavior", IEEE Transactions on Smart Grid, 2(2), pp. 302-313 (2011).

26. Rüther, R., Pereira Junior, L.C., Bittencourt, A.H., Drude, L., and Dos Santos, I.P. "Strategies for plug-in electric vehicle-to-grid (V2G) and photovoltaics (PV) for peak demand reduction in urban regions in a smart grid environment", In Plug In Electric Vehicles in Smart Grids, S. Rajakaruna et al., Springer, Singapore (2015).

27. Rosenkranz, K. "Deep-cycle batteries for plug-in hybrid application", 20th Electric Vehicles Symp. (EVS 20), Long Beach, CA (2003).

28. "Preliminary regulation summary", January 2013, Report by PJM (2013). Available at: http://www.pjm.com, accessed November 2013.
29. "PJM manual 28: Operating agreement accounting, revision 71, June 1, 2015", Report by PJM (2015). Available at: http://www.pjm.com/marketsand-operations /ancillary services.aspx, accessed December 2015 .

30. "Reg-data-external-1-31-January-2013", Report by PJM (2013). Available at: http://www.pjm.com, accessed November 2013.

31. Pavić, I., Capuder, T., and Kuzle, I. "Value of flexible electric vehicles in providing spinning reserve services", Applied Energy, 157, pp. 60-74 (2015).

32. "Stem CTO: Lithium-Ion battery prices fell $70 \%$ in the last 18 months", Report by S. Lacey, Available at: https://www. greentechmedia.com/articles/read/ stem-cto-weve-seen-battery-prices-fall-70-in-the-last18-months, accessed January 2017.

\section{Biographies}

Mohammad Hossein Sarparandeh received BSc and MSc degrees in Electrical Engineering from Sharif University of Technology, Tehran, Iran in 2008 and 2010, respectively. He is currently pursuing the $\mathrm{PhD}$ degree at the Department of Electrical Engineering, Sharif University of Technology, Tehran, Iran. His research interests include operation \& planning of power systems, microgrids, and power markets.

Mostafa Kazemi received the $\mathrm{BSc}, \mathrm{MSc}$, and $\mathrm{PhD}$ degrees in Electrical Engineering from Sharif University of Technology, Tehran, Iran in 2010, 2012, and 2016, respectively. He is currently with the Department of Electrical Engineering, University of Shahreza, Shahreza, Iran. His research interests include economics and planning of restructured power systems.

Mehdi Ehsan received the BSc and MSc degrees in Electrical Engineering from Technical College of Tehran University, Tehran, Iran in 1963 and the PhD and DIC degrees from Imperial College University of London, London, U.K. in 1976. He currently is a Full Professor at the Department of Electrical Engineering, Sharif University of Technology, Tehran and The Center of Excellence in Power System Management and Control. His research interests are restructuring, planning \& control of power systems as well as power system dynamics. 